\title{
ORGANIZACIONES EMOCIONALMENTE INTELIGENTES
}

\section{EMOTIONALLY INTELLIGENT ORGANIZATIONS}

\author{
Paloma del Henar Sánchez Cobarro ${ }^{1}$ Universidad de Murcia. España.
} palomahenar.sanchez@um.es

\section{RESUMEN}

Las investigaciones en inteligencia emocional han puesto de manifiesto la repercusión que los aspectos emocionales tienen en la vida de los individuos y han cuestionado que sean válidas por sí solas las mediciones de la inteligencia cognitiva tal y como se ha pretendido durante decenios. Desde que en 1995 se popularizara y se diera a conocer fuera del ámbito científico el concepto "inteligencia emocional" se ha experimentado sobre este campo un considerable interés en el terreno académico y organizacional, y se han incrementado las investigaciones al respecto. Se trata por tanto de un ámbito de estudio en constante evolución.

PALABRAS CLAVE: razón, emoción, inteligencia, organizaciones, empresas, autoconocimiento, recursos humanos.

\section{ABSTRACT}

Research on emotional intelligence have highlighted the impact that emotional aspects have on the peoples' life, and have questioned the validity of the measurements of cognitive intelligence. Since the term "emotional intelligence" became known in the scientific field back in 1995, this sphere has received a lot of interest from both the academic and the organizational areas, and the investigations regarding it have increased. Therefore, it is a constantly developing field of study.

KEY WORDS: reason, emotion, intelligence, organizations, businesses, selfawareness, human resources.

\section{Cómo citar el artículo:}

del Henar Sánchez Cobarro, P. (2016). Organizaciones emocionalmente inteligentes. Revista de Ciencias de la Comunicación e Información, 21(1), 19-33. doi: http://doi.org/10.35742/rcci.2016.21(1).19-33

\section{INTRODUCCIÓN. INTELIGENCIA: RAZÓN Y EMOCIÓN}

Desde el momento en que el hombre empieza a tomar conciencia de los componentes emocionales que acompañan su existencia, comienza a mantener una

\footnotetext{
1 Paloma del Henar Sánchez Cobarro: Doctora en CC. de la Comunicación. Profesora de la Universidad de Murcia. Consultora de comunicación. Especialista en recursos humanos e inteligencia emocional. Universidad de Murcia, España.
} 
constante lucha entre los sentimientos y la razón, que fraguan su comportamiento y sus actitudes ante la vida.

Se ha intentado demostrar durante años que las personas están condicionadas por su inteligencia, prestando interés únicamente a la valoración de los componentes racionales en un intento por evaluar, con ayuda de distintos métodos, las capacidades intelectuales de los individuos.

Este interés que continúa hoy vigente, tiene unos antecedentes no muy lejanos, según Rafael Bisquerra "la investigación sobre la inteligencia probablemente se inicia con los estudios de Broca (1824-1880), que estuvo interesado en medir el cráneo humano y sus características, y que llegó a descubrir la localización del área del lenguaje en el cerebro. Al mismo tiempo, Galton (1822-1911) bajo la influencia de Darwin, realizaba sus investigaciones sobre los genios, aplicando la campana de Gauss. También en esta época Wundt (1832-1920) estudiaba los procesos mentales mediante la introspección. Pero es a partir de Binet cuando se habla de la medición de la inteligencia cuyos efectos sobre la educación son imponderables" (Bisquerra, 2003, p. 14).

El interés por averiguar el cociente intelectual para llegar a medir la inteligencia de los individuos, comenzó a hacerse más patente a partir del siglo XX. Los primeros investigadores que llevaron a cabo un método de evaluación, fueron Alfred Binet y Théodore Simon, dejando constancia en 1905 del primer test de medición de la inteligencia. Aplicaron esta técnica por petición del Ministerio de Educación francés en distintas escuelas de París, con el objetivo de poder identificar a aquellos niños que por poseer un coeficiente intelectual inferior al establecido, debían recibir clases de educación especial.

Unos años más tarde, en 1912, William Stern introdujo el término coeficiente intelectual $(\mathrm{Cl})$. Este psicólogo alemán formuló una escala de medición del $\mathrm{Cl}$, según la cual, relacionando la edad mental y la cronológica de una persona, se obtiene el nivel de inteligencia que ésta posee.

Poco tiempo después, en 1916, el psicólogo de la Universidad de Stanford, Lewis Terman, aplicó la escala establecida por Binet y Simon a niños en Estados Unidos, por lo que pasó a denominarse escala de inteligencia Stanford-Binet.

Pero el hecho que popularizó el trabajo de este psicólogo, por el que se le reconoce y algunos llegan a considerarlo el creador de los tests de inteligencia que determinan nuestro coeficiente intelectual, fue que durante la I Guerra Mundial, ayudó a reclutar para el ejército norteamericano, a dos millones de varones aplicando los tests de inteligencia. Años más tarde, en 1937 y 1960, se llevaron a cabo revisiones de este instrumento de medición que, aumentaron aún más su popularidad y extendieron el término $\mathrm{Cl}$ a la sociedad. James McKenn Cattell difundió los tests de inteligencia en Estados Unidos gracias a sus investigaciones basadas en el estudio de diferencias entre individuos, apoyándose en las respuestas que éstos daban a los tests. Posteriormente Charles Spearman y Louis Thurstone aplicaron el análisis factorial al estudio de la inteligencia. 
Thurtone estableció en 1938 que existen siete habilidades mentales primarias: comprensión verbal, fluidez verbal, capacidad para el cálculo, rapidez perceptiva, representación espacial, memoria y razonamiento inductivo. A partir de este enfoque factorial, Guilford, llevó a cabo en 1950 sus trabajos sobre estructura de la inteligencia, que extendieron el área de aplicación al estudio de la creatividad y el pensamiento divergente.

Años más tarde, Howard Gardner, psicólogo de la Universidad de Harvard, propone una teoría en la que plantea la existencia de inteligencias múltiples (1983). Este autor publica Multiple intelligences. Thetheory in practiceen 1993, dos años después se traduce al castellano. En esta obra propone un conjunto de inteligencias múltiples que coexisten y actúan de manera independiente, niega la existencia de una inteligencia general y única, y le otorga una especial relevancia a la evaluación de las inteligencias múltiples en el ámbito educativo.

En Inteligencias múltiples. La teoría en la práctica, Gardner distingue siete categorías de inteligencia: cinético-corporal, lógico-matemática, lingüística, espacial, musical, interpersonal e intrapersonal.

Es a partir de este momento cuando comienzan a considerarse las distintas capacidades que posee un individuo y no tanto la medición cuantitativa de la inteligencia. La consideración, por parte de Gardner, de la inteligencia interpersonal e intrapersonal supone un acercamiento a la inteligencia emocional y por supuesto, forman parte del cuerpo teórico de la misma.

A partir de entonces se empieza a tomar conciencia de que, aunque los tests de inteligencia valoren nuestra capacidad racional, el resultado que ofrecen no puede demostrar la inteligencia global que posee un individuo, ya que sólo constituye una aproximación parcial a la capacidad de razonamiento.

Las personas no sólo disponen de una capacidad racional, sino que también existe un componente emocional que guía sus acciones $y$, por tanto, se encuentran influidas por ambas. De ahí que resulte necesario investigar también acerca de cómo los sentimientos y las pasiones conducen a los individuos, orientando, condicionando o determinando sus comportamientos.

Ante esta necesidad y ante la creencia de que los individuos son emocionalmente inteligentes, surge el estudio de la inteligencia emocional, que constituye otro modo de intentar valorar la capacidad emocional que poseen, y así potenciarla a través del conocimiento de ellos mismos y de su modo de relacionarse con los demás, en la búsqueda de la felicidad y el equilibrio.

En este sentido entendemos la IE como una capacidad propia del ser humano que puede ser desarrollada a medida que éste evoluciona en el conocimiento de sí mismo y de su entorno, reconociendo no sólo sus propios estados emocionales sino también los ajenos. En definitiva, el estudio de la IE se basa en el desarrollo de habilidades emocionales que permiten al individuo un dominio lo más adecuado posible de sí mismo y sus capacidades. 
Por ello es fundamental contemplar la IE en de los individuos como un elemento primordial a tener en cuenta en el desarrollo de habilidades, capacidades y competencias necesarias tanto en el ámbito profesional, como personal y social.

En 1995 Daniel Goleman, con la publicación de su obra Inteligencia emocional, popularizó el término, que hasta ese momento sólo había sido desarrollado y expuesto en el entorno de las investigaciones de la comunidad científica. Esta obra que se convirtió en un best-seller tuvo una gran influencia, no sólo porque acercó un término hasta entonces desconocido, sino porque a ella le sucedieron otras investigaciones, se dieron a conocer las realizadas hasta el momento y se comenzó a aplicar con más intensidad el estudio de la inteligencia emocional a diversos campos, como la educación, la autoayuda, el entorno laboral, etc.

\section{OBJETIVOS}

Profundizar en la relación de la inteligencia emocional con el entorno empresarial, concretamente con el desarrollo de habilidades, capacidades y competencias de los profesionales y su conceptualización y aplicación en el trabajo.

\section{METODOLOGÍA}

Atendiendo al estado de conocimiento del tema y al enfoque que se pretende dar a este estudio será explicativo y descriptivo. Se trata de un artículo de corte ensayístico. Se ha constituido bajo una base metodológica fundamentalmente teórica. Se ha recurrido a la revisión bibliográfica y hemerográfica, para construir la base documental que nos ha permitido establecer cuáles son los fundamentos de la inserción de la inteligencia emocional en las organizaciones.

\subsection{Antecedentes de la inteligencia emocional}

Hay autores que realizan un acercamiento al concepto de inteligencia emocional desde la teoría de las emociones, remontándose de este modo a los orígenes de la filosofía y la literatura, estableciendo relaciones entre la búsqueda del bienestar y la felicidad desde la Antigüedad clásica. Pero, puesto que hay evidencias que demuestran que los orígenes claramente científicos de la inteligencia emocional se encuentran a partir del siglo XIX, abordaremos la aproximación al constructo desde esa determinación.

Comenzando por el británico Charles Darwin que centró sus investigaciones en el desarrollo de la teoría de la evolución. En su obra "El origen de las especies" (Darwin, 2003) publicada en 1859, destacaba que desde sus orígenes el hombre ha desarrollado el manejo de las emociones con el propósito de prepararse para la acción, manifestándose de forma más explícita en las situaciones de peligro o emergencia. Desde una perspectiva similar, Darwin publicó en 1876 la obra "La expresión de las emociones en el hombre y los animales" (Darwin, 1984). Esta obra constituye una investigación en la que se relaciona la conducta de los animales y la humana, poniendo de manifiesto la proximidad existente entre los monos y los hombres en lo que a la expresión de emociones se refiere. De este modo estableció una relación entre las emociones y los componentes biológicos, ya que mediante el 
método de observación pudo constatar que existen actos animales similares a hábitos expresivos humanos.

Las primeras propuestas sobre el estudio de la emoción deben ser atribuidas a Charles Darwin, William James y Walter B. Cannon, aunque después de sus hallazgos la investigación sobre las emociones sufrió un estancamiento hasta la llegada de la investigación científica de la emoción con la psicología cognitiva.

Se tendrá que llegar al siglo XX, para encontrar la definición de inteligencia emocional, tal y como la conocemos hoy, y así, poder delimitar aquellos componentes que forman parte de su estructura.

A comienzos de ese siglo, concretamente en 1920, L. Thorndike profesor de psicología educativa de la Universidad de Columbia, a quien algunos autores señalan como precursor del concepto inteligencia emocional, estableció el término inteligencia social para referirse a ciertas capacidades que poseen los individuos. La definió como la "habilidad para entender y manejar a los hombres, mujeres, muchachos y para actuar sabiamente en las relaciones humanas" (Vallés Arándiga y Vallés Tortosa, 2003, p. 27). Thorndike destacaba de la inteligencia social que "era una habilidad que se mostraba en las guarderías, en el patio de recreo, en los cuarteles y en las fábricas y era difícil observarla en las situaciones formales del laboratorio" (Vallés Arándiga y Vallés Tortosa, 2003, p. 27). La definición de inteligencia social de este autor, permite ya entrever e identificar algunos de los aspectos característicos de la inteligencia emocional.

A las aproximaciones de L. Thorndike les seguiría un periodo dominado por las teorías conductistas de autores como John B. Watson (1878-1958) y B. F. Skinner (1904-1990), que cobraron importancia durante las décadas cuarenta y cincuenta.

Watson, fundador de la escuela de psicología conductista, plantea la importancia de analizar el comportamiento externo de los individuos, y como aportación a la psicología propone que no sólo se centre en el estudio de la estructura mental del ser humano sino también en la conducta, aplicando métodos objetivos de análisis.

Por su parte, Skinner fue el encargado de ampliar la teoría de Watson introduciendo en la psicología académica el conductismo más radical, entre otras razones porque a diferencia de Watson, consideraba que debían ser excluidos en el estudio del ser humano todos los fenómenos que forman parte de su estructura interna, tales como los sentimientos y las emociones. De este modo quedarían fuera de su análisis aquellos componentes de la personalidad que, a pesar de ser inherentes en el ser humano, no pueden ser medidos de modo objetivo a través de métodos de observación.

Por lo tanto, fueron excluidos todos los aspectos relacionados con las emociones de los individuos. Es decir, el conductismo con Skinner redujo el análisis del comportamiento humano a procesos de estímulo-respuesta, observables y medibles. Posteriormente, a finales de la década sesenta, se produce una ruptura con la teoría conductista y surge el cognitivismo que abandona toda creencia basada en la pasividad del individuo y en su comportamiento mecanicista. El cognitivismo bajo la 
premisa de que los individuos actúan sobre la base de sus creencias, actitudes y deseos para alcanzar ciertas metas sin ser guiados por estímulos, revolucionó la psicología aplicada hasta el momento. Se produjo un cambio de dirección que condujo a que los sentimientos, las percepciones y la motivación interna fueran los elementos primordiales que guiaran la vida del ser humano. A partir de entonces, se produjo el intento de averiguar cómo la mente registra y almacena la información y cuál es la naturaleza de la inteligencia. La teoría cognitivista se centró en aspectos como el procesamiento de la información por parte de los seres humanos, pero sin tener en cuenta el lugar que ocupan las emociones y los sentimientos en la mente de los individuos.

Conforme avanzan las investigaciones en psicología y el intento por conocer al ser humano, se van contemplando todos los elementos que forman parte de su capacidad emocional, pero fueron estas dos corrientes, el conductivismo y el cognitivismo, las que se convirtieron en las teorías dominantes de la psicología experimental del siglo XX.

Aun así no habría que esperar mucho tiempo para que el término inteligencia emocional apareciera escrito en una obra. En 1966, Jane Austen recurre por primera vez al concepto de inteligencia emocional en un libro de crítica literaria en el que mencionaba "una inteligencia que informa a las emociones" (Vallés Arándiga y Vallés Tortosa, 2003: 27). Ese mismo año, B. Leuner publicó "un artículo en alemán cuya traducción sería Inteligencia emocional y emancipación. En él se planteaba el tema de cómo muchas mujeres rechazan un rol social a causa de su baja inteligencia emocional" (Bisquerra, 2003, p. 16); a pesar de esta aproximación, en estos estudios el término de inteligencia emocional todavía no aparecía desarrollado. Howard Gardner, publicóen 1983 Frames of Mind: The Theory of Multiple Intelligences. En esta obra propone diferentes tipos de inteligencia que seguirán facilitando el camino hacia la consecución del término IE.

Bajo esta misma perspectiva, Robert Sternberg publicará en 1985, Beyond IQ: A triarchictheory of human intelligence, obra en la que desarrolla su teoría acerca de las diferentes inteligencias que están presentes en el ser humano y que conducirá años más tarde a la implantación, por parte de este autor, del concepto inteligencia exitosa.

En 1985, Wayne Payne publicó su tesis doctoral bajo el título: "A study of emotion: developingemotionalintelligence"; fue esta la primera vez que el concepto inteligencia emocional aparecía en el entorno académico.

En 1988, Reuven Bar-On presentó su tesis doctoral Thedevelopment of a concept of psychologicalwell-being (Bar-On 1988), convirtiéndose en precedente del estudio de la inteligencia al proponer un cociente emocional (EQ) paralelo al coeficiente intelectual. Para este autor tuvieron una gran influencia las investigaciones llevadas a cabo por la psicóloga Marie Jahoda, que relacionó la salud mental con el bienestar psicológico, con la intención de demostrar la influencia que éste último podía llegar a ejercer sobre el primero y restándole importancia a los estudios psicopatológicos. Está autora estudió componentes de bienestar psicológico que fueron la base sobre la que Bar-On estableció los siguientes factores de personalidad relacionados con el 
bienestar psicológico: la autoobservación, las relaciones interpersonales, la responsabilidad social, la flexibilidad, la independencia, la resolución de problemas, la asertividad, la prueba de realidad, la tolerancia al estrés, la actualización y la felicidad. A partir de estos factores Bar-On construye su inventario de inteligencia emocional (EQ-I) y comienza un periodo en el que en psicología cobran importancia el estudio de la personalidad y los factores determinantes de la felicidad personal. Por ello los trabajos realizados por Bar-On desde su tesis doctoral, suponen los primeros estudios llevados a cabo sobre inteligencia emocional.

Sin embargo, el interés por la inteligencia emocional lo despertaron dos artículos de Salovey y Mayer publicados en 1990. "En ellos repasaron las investigaciones existentes en las áreas de la psicología y la psiquiatría, la inteligencia artificial y otras áreas. De dicho análisis hipotetizaron la existencia de una habilidad humana que bien pudiera denominarse inteligencia emocional. Para ello se basaron en la idea de que unas personas razonaban mejor que otras sobre las emociones y también en la idea de que ese razonamiento emocional se veía mejorado por estados emocionales positivos adecuados. Como consecuencia de ello, presentaron la primera definición formal de IE, a la vez que apuntaron que determinadas tareas de habilidad podrían servir para medir dicho constructo. A pesar de ello, estos autores no se atribuyen el constructo de IE, sino que a ella se ha llegado paulatinamente" (Vallés Arándiga y Vallés Tortosa, 2003, p. 30).

El impulso al concepto inteligencia emocional y el reconocimiento fuera de la comunidad científica es atribuido a Goleman, psicólogo de la Universidad de Harvard, quien en su obra Inteligencia emocional, realiza aproximaciones, propias y ajenas a la delimitación del término, definiéndolo desde diferentes perspectivas y aportando las conclusiones de aquellos autores que han llevado a cabo investigaciones al respecto, ayudando a la configuración del concepto.

\subsection{Del concepto al modelo}

Las investigaciones sobre inteligencia emocional llevadas a cabo en los últimos años han centrado gran parte de sus esfuerzos en construir su corpus epistemológico. Este interés por evaluar la inteligencia emocional provocó desde un principio que las aproximaciones al concepto se realizaran desde diversas perspectivas teóricas, lo que trajo consigo que, a su vez, en el desarrollo de los modelos de inteligencia emocional estén presentes distintas consideraciones, dependiendo de la base teórica sobre la que se fundamenta cada investigación.

Entre los distintos modelos de inteligencia emocional se pueden identificar dos tendencias: la primera de ellas se corresponde con lo que se ha denominado modelos mixtos y la segunda con los modelos de habilidades.

Respecto a los modelos mixtos, éstos incluyen componentes procedentes de la personalidad, "rasgos como el control del impulso, la motivación, la tolerancia a la frustración, el manejo del estrés, la ansiedad, el optimismo, la asertividad, la confianza, la persistencia, etc., parecen solaparse con componentes emocionales y con estilos de comportamiento socio-emocional" (Vallés Arándiga y Vallés Tortosa, 2003: 37). También se identifican, estos modelos, por pretender un conocimiento 
que sea a la vez directo y aplicativo, no únicamente teórico. Alguno de los investigadores que han desarrollado modelos mixtos son: Daniel Goleman, Reuven Bar-On, R. K Cooper y A. Sawaf.

Los modelos de habilidades, al contrario de los anteriores no incluyen factores de la personalidad, "postulan la existencia de una serie de habilidades cognitivas o destrezas para evaluar, expresar, manejar y autorregular la emocionalidad de un modo inteligente y adaptado al logro del bienestar personal, teniendo en cuenta las normas sociales y los valores éticos" (Vallés Arándiga y Vallés Tortosa, 2003, p. 4243). El modelo de habilidades con mayor reconocimiento en la comunidad científica es el desarrollado por Peter Salovey y John Mayer.

Las diferencias fundamentales entre los modelos mixtos y los modelos de habilidades se pueden resumir de la siguiente manera: mientras los primeros combinan factores de la personalidad con el desarrollo de habilidades emocionales, los modelos de habilidades se centran en el estudio de las capacidades presentes en el procesamiento de la información emocional.

Los modelos de inteligencia emocional con mayor reconocimiento en la comunidad científica coinciden en considerar el conocimiento emocional -cada uno con su propia denominación- un factor fundamental para alcanzar el desarrollo que permite a un individuo ser emocionalmente inteligente. Aunque cada modelo proponga una estructura y aplique una terminología diferente al resto, todos se sustentan en los dos pilares fundamentales de la inteligencia emocional: la inteligencia intrapersonal y la inteligencia interpersonal. Todos proponen un crecimiento interior que finalmente se refleja en la forma en que un individuo percibe y entiende a los demás.

En cualquier relación personal o profesional están presentes los dos componentes fundamentales de la inteligencia emocional: la inteligencia intrapersonal y la inteligencia interpersonal.

La primera de ellas "constituye una habilidad correlativa -vuelta hacia el interiorque nos permite configurar una imagen exacta y verdadera de nosotros mismos y que nos hace capaces de utilizar esa imagen para actuar en la vida de un modo más eficaz". Por otra parte, la inteligencia interpersonal "consiste en la capacidad de comprender a los demás: cuáles son las cosas que más les motivan, cómo trabajan y la mejor forma de cooperar con ellos" (Gardner, Howard, 1993, p. 9). La inteligencia intrapersonal está presente en el entrevistador y en el entrevistado. Es la que facilita a los individuos el ser capaces de reconocer sus estados emocionales y así poder identificar las emociones que experimenta la persona que está junto a ellos, habilidad imprescindible en procesos de negociación, por ejemplo.

Por su parte, las investigaciones in inteligencia emocional han demostrado que la inteligencia interpersonal ayudará a que los individuos se relacionen adecuadamente con otras personas, entendiendo su comportamiento y empatizando con ellas. Por ello en las relaciones profesionales cobra una relevancia especial este tipo de inteligencia.

El desarrollo de competencias de inteligencia emocional en cualquier campo profesional resulta imprescindible, ya que todo proceso comunicativo se ve influido 
por la inteligencia intrapersonal y supone una puesta en práctica de la inteligencia interpersonal. La interacción verbal como base del trabajo requiere habilidades de los profesionales que, en unos casos se habrán desarrollado de forma innata mientras en otros, deberán ser aprendidas.

\section{RESULTADOS}

Todos los individuos poseen unas determinadas capacidades emocionales innatas. Éstas no tienen por qué ser las mismas en todas las personas ni estar desarrolladas en la misma medida, pues dependen de las cualidades personales, conocimiento, experiencia y madurez de los individuos. Pero para la consecución de competencia emocional, es necesario que las capacidades emocionales de los profesionales (innatas y adquiridas) se pongan en práctica, es decir, se manifiesten como habilidades emocionales. El desarrollo de estas habilidades contribuye a un conocimiento más exhaustivo de las propias emociones, lo que implica el poder regularlas hacia los objetivos deseados.

Entre los autores que definen la inteligencia emocional como una capacidad, podemos encontrar a Caruso, Goleman, Cooper y Sawaf, entre otros.

Sin embargo, hay quienes la identifican como una habilidad, tal es el caso de Salovey y Mayer; y otros, finalmente, como Bar-On, definen la inteligencia emocional como un "conjunto de capacidades, competencias y habilidades no cognitivas que influencian la habilidad propia de tener éxito al afrontar las demandas y presiones del medio ambiente" (Bar-On, 1997). Este autor incluye los tres conceptos en su definición pero no delimita la función de cada uno de ellos.

Al hablar de competencias, es preciso en primer lugar hacer referencia a David McClelland, quien a principios de los años setenta comenzaba a ser conocido por sus estudios sobre la motivación laboral. Este autor sostenía que tanto los tests de aptitudes como los de conocimientos aplicados tradicionalmente no eran válidos como predictores del éxito de un individuo. Además, consideraba que eran sesgados, pues discriminaban por razones de sexo, raza o nivel socioeconómico. Por ello comenzó a buscar otros componentes que debían ser considerados en la predicción del éxito laboral; esto le condujo a la discriminación de aspectos individuales y a combinar las habilidades, conocimientos y atributos personales que posee un individuo. De este modo estableció lo que vino a llamar, competencias.

Desde una perspectiva similar, años más tarde, Daniel Goleman discípulo de McClelland, continúa insistiendo en la importancia de las competencias en el entorno laboral, pero las denominará competencias emocionales. Según este autor en el nuevo marco laboral que se está gestando en las empresas, "con su énfasis en la flexibilidad, los equipos y la fuerte orientación hacia el servicio, las competencias emocionales resultarán cada vez más decisivas para alcanzar la excelencia en cualquier trabajo y en cualquier país del mundo" (Goleman, Daniel, 1999, p. 52).

Goleman define la competencia emocional como "una capacidad adquirida basada en la inteligencia emocional que da lugar a un desempeño laboral sobresaliente", y asegura que nuestra inteligencia emocional "determina la capacidad potencial de 
que dispondremos para aprender las habilidades prácticas basadas en uno de los siguientes cinco elementos compositivos: la conciencia de uno mismo, la motivación, el autocontrol, la empatía y la capacidad de relación. Nuestra competencia emocional, por su parte, muestra hasta qué punto hemos sabido trasladar este potencial a nuestro mundo laboral" (Goleman, Daniel, 1999, p. 52).

Este autor matiza en relación con las competencias emocionales que éstas "se agrupan en conjuntos, cada uno de los cuales está basado en una capacidad subyacente de la IE, capacidades que son vitales si las personas quieren aprender las competencias necesarias para tener éxito en su trabajo" (Goleman, Daniel, 1999, p. 47) e identifica como capacidades de la inteligencia emocional las siguientes:

- Independencia: cada persona aporta una contribución única al desempeño de su trabajo.

- Interdependencia: cada individuo depende en cierta medida de los demás, con los que se halla unido por interacciones muy poderosas.

- Jerarquización: las capacidades de la IE se refuerzan mutuamente.

- Necesidad pero no suficiencia: poseer una IE subyacente no garantiza que la gente acabe desarrollando o ejerciendo las competencias asociadas.

- Genéricas: la lista general resulta, hasta cierto punto, aplicable a todos los trabajos, pero cada profesión exige competencias diferentes.

Antonio Vallés Arándiga y Consol Vallés Tortosa sostienen que "las competencias emocionales están constituidas por destrezas, habilidades y comportamientos de automanejo y de interacción con los demás y con el entorno" (Vallés Arándiga y Vallés Tortosa, 2003: 37-38).

Para CarolynSaarni la competencia emocional es "la demostración de autoeficacia en expresar emociones en las transacciones sociales (emotion-elicitingsocial transaccions). Autoeficacia significa que el individuo cree que tiene la capacidad y las habilidades para lograr objetivos deseados.

Si bien es cierto que todos podemos experimentar incompetencia emocional en un momento dado y en un espacio determinado, dado que no nos sentimos preparados para esa situación" (Bar-On y Parker, 2000, p. 68).

Que los individuos manifiesten sus emociones de una forma controlada ha sido durante años la educación recibida y el comportamiento socialmente aceptado. Las emociones han sido vistas como obstáculos para nuestras relaciones por lo que debían ser reprimidas. Y esta afirmación se pone especialmente de manifiesto en el entorno laboral, donde es considerado poco profesional la expresión de emociones tanto positivas como negativas, por lo que se fomenta un clima emocionalmente neutro.

Y esto, a pesar de que las emociones son absolutamente decisivas en la toma de decisiones, la resolución de conflictos, las relaciones interpersonales, etc.

Por todo ello consideramos que en las relaciones laborales, deben estar basadas en el entendimiento entre los miembros de una organización, y ello, sólo es posible a través de la comprensión del comportamiento emocional propio y ajeno. De ahí que 
para contribuir a un clima laboral óptimo, consideramos preciso recurrir a una estructura sólida de desarrollo de la inteligencia emocional, tal y como proponen Salovey y Mayer, en su modelo científico.

El modelo desarrollado por estos autores se centra en un conjunto de habilidades relacionadas con el procesamiento de la información emocional y el estudio de las capacidades relacionadas con dicho procesamiento.

Fernández-Berrocal y Extremera consideran que la teoría desarrollada por Mayer y Salovey es la más extendía y avalada empíricamente. Desde la teoría que propone este modelo, la inteligencia emocional es definida como "la habilidad de las personas para atender y percibir los sentimientos de forma apropiada y precisa, la capacidad para asimilarlos y comprenderlos de manera adecuada y la destreza para regular y modificar nuestro estado de ánimo y el de los demás" (Fernández-Berrocal y Extremera, 2002, p. 1-6).

Este modelo está formado por cuatro etapas, cada una de las cuales implica el desarrollo de un componente emocional. Las habilidades que integran cada una de estas etapas son superadas a medida que se avanza en las etapas que constituyen el modelo.

Por ello, el primer elemento que tienen en cuenta estos autores es la percepción emocional, ya que es el nivel más básico en el desarrollo de habilidades, durante la cual los individuos toman conciencia de su capacidad emocional, lo que les permite identificar sus propias emociones y las de los demás. Una vez que se ha desarrollado esa habilidad es necesario manejar esas emociones hacia una finalidad, favoreciendo la toma de decisiones y la resolución de los problemas, lo que Salovey y Mayer identifican como facilitación emocional.

Lo aprendido anteriormente permite que los individuos desarrollen su comprensión emocional, que es la que les proporcionará entender el funcionamiento de las emociones y los distintos estados emocionales que éstas generan. Por último, la regulación emocional supone el control de las emociones tanto positivas como negativas, la normalización de las mismas, y por tanto, el equilibrio que otorga reconocer nuestros estados emocionales entendiendo así, también los ajenos.

Salovey y Mayer no proponen en su modelo el desarrollo de habilidades sociales, ni contemplan factores de la personalidad. Han creado un modelo cuya finalidad principal es que los individuos lleguen a alcanzar la regulación emocional y el crecimiento personal. 


\begin{tabular}{|c|l|}
\hline Habilidades integrantes & Breve descripción \\
\hline - Percepción emocional & $\begin{array}{l}\text { La habilidad para percibir las propias } \\
\text { emociones y las de los demás, así como } \\
\text { percibir emociones en objetos, arte, historia, } \\
\text { música y otros estímulos. }\end{array}$ \\
\hline - Facilitación emocional & $\begin{array}{l}\text { La habilidad para generar, usar y sentir las } \\
\text { emociones como necesarias para comunicar } \\
\text { sentimientos, o utilizarlas en otros procesos } \\
\text { cognitivos. }\end{array}$ \\
\hline - Comprensión emocional & $\begin{array}{l}\text { La habilidad para comprender la información } \\
\text { emocional, cómo las emociones se combinan y } \\
\text { progresan a través del tiempo y saber apreciar }\end{array}$ \\
los significados emocionales.
\end{tabular}

Fuente: N. Extremera y P. Fernández-Berrocal, 2004.

Tanto el modelo de habilidades de Mayer y Salovey como los modelos mixtos presentan, a pesar de sus diferencias, rasgos comunes. Todos los investigadores presentes en estos modelos consideran que el cociente intelectual no es un valor suficiente a tener en cuenta para garantizar el éxito en la vida; es necesario desarrollar habilidades, capacidades y competencias emocionales que permitan a los individuos manejar sus propias emociones e identificar y reconocer las de los demás.

Es por ello que todos persiguen que los individuos sean capaces de utilizar de forma adaptativa las emociones, manejar y regular sus estados emocionales e identificar tanto sus emociones como las de los demás.

En este sentido, Rafael Bisquerra establece una estructuración de competencias emocionales, entre las que destaca, en primer lugar, la conciencia emocional. La define como la "capacidad para tomar conciencia de las propias emociones y de las emociones de los demás, incluyendo la habilidad para captar el clima emocional de un contexto determinado" (Bisquerra, 2003, p. 23).

De la propuesta de Bisquerra podemos destacar que vincula ambos territorios, intrapersonal e interpersonal, en una misma definición. No concibe construir el concepto de conciencia emocional sin mencionar la repercusión que supone poseer esta competencia en las relaciones con los demás.

En definitiva, podemos considerar el autoconocimiento como una competencia que permite a los individuos comprender sus emociones, identificarlas y diferenciarlas. 
Es un proceso auto-reflexivo a través del cual los individuos toman conciencia de su yo personal, de las características que le identifican y que constituyen su individualidad. Ello implica realizar ejercicios de auto-percepción, auto-observación y auto-aceptación, es decir, realizar un proceso de autoevaluación que permite a los profesionales identificar sus debilidades y fortalezas y emprender un aprendizaje hacia áreas de mejora. De este modo, se refuerza la autoestima y la confianza en uno mismo, lo que genera un control emocional que aporta beneficios personales que se extrapolan al desempeño de la profesión. En este sentido, Goleman, Boyatzis y McKee (Boyatzis, McKee y Goleman, 2002) al establecer las competencias relacionadas con el liderazgo insisten en que la autoevaluación debe ser un proceso imprescindible para realizar el correcto autoconocimiento emocional.

Daniel Goleman asegura que las investigaciones realizadas a nivel mundial por decenas de expertos, en cerca de quinientas empresas, agencias gubernamentales y organizaciones no lucrativas parecen coincidir en subrayar el papel determinante que juega la inteligencia emocional en el desempeño óptimo de cualquier tipo de trabajo.

Por último, nos gustaría destacar que en esta investigación consideramos que las competencias de inteligencia emocional pueden y deben desarrollarse. "No obstante, el desarrollo requiere tiempo, compromiso y apoyo. Además, las empresas suelen impedir más que facilitar el proceso de desarrollo. El énfasis en producir resultados inmediatos suele dar paso a métodos de desarrollo coercitivos o poco inteligentes emocionalmente, que a su vez refuerzan los malos comportamientos y que tienen un precio a la larga en la salud de la organización y de sus miembros. Incluso en las numerosas organizaciones que proporcionan formación normalmente, el modelo de dicha formación suele estar diseñado para producir un cierto nivel de habilidades técnicas o cognitivas" (Goleman y Cherniss, 2005, pp. 248-249).

\section{CONCLUSIONES Y DISCUSIÓN}

El concepto de inteligencia a pesar de los años que lleva de desarrollo, investigación y puesta en práctica es un concepto relativamente reciente en el mundo laboral, yha sido en estos últimos años cuando las organizaciones más se han interesado por la formación y el desarrollo de la inteligencia emocional de sus empleados, aun así, todavía quedan grandes campos sin explorar y tanto en salud, como en educación como en el tejido empresarial se siguen detectando carencias emocionales que afectan al comportamiento individual y colectivo.

Goleman y Cherniss, principalmente, han demostrado cómo incluir aspectos de inteligencia emocional en la gestión empresarial repercute positivamente en la gestión de las personas y los recursos, lo que a su vez influye en los beneficios económicos de las organizaciones.

El desarrollo y puesta en práctica de competencias emocionales ha despertado el interés de investigadores, docentes, directivos y gerentes en estas últimas décadas y ha ayudado a sentar bases para su aplicación en la búsqueda del bienestar personal y profesional. 


\section{REFERENCIAS}

Bar-On, R. (2006). The Bar-On model of Emotional-Social Intelligence. Psicothema, 18(1), 13-25.

Bar-On, R. (1997). The Emotional Quotient Inventory (EQ-I): Technical Manual, Multi-Health Systems, Canadá.

Bar-On, R. y A. Parker, J. D. (Eds.) (2000). The Handbook of Emotional Intelligence. Theory, Development, Assessment, and Application at Home, School, and in the Workplace, San Francisco, Ca: Jossey-Bass.

Bisquerra, R. (2000). Educación emocional y bienestar. Barcelona: Praxis.

Bisquerra, R. (2003). Educación emocional y competencias básicas para la vida. Revista de Investigación Educativa (RIE), 21(1), 7-43.

Boyatzis, R., McKee, A. y Goleman, D. (2002). El líder resonante crea más: el poder de la inteligencia emocional. Barcelona: Plaza \& Janés,

Darwin, C. (1984). La expresión de las emociones en los animales y en el hombre. Madrid: Alianza.

Gardner, H. (1995). Inteligencias múltiples. La teoría en la práctica. Barcelona: Paidós.

Gardner, H. (2001). La inteligencia reformulada. Las inteligencias múltiples en el siglo $X X I$. Barcelona: Paidós.

Gardner, H. (1993). Multiples Intelligences. New York: Basic Books.

Goleman, D. (1999). La práctica de la Inteligencia Emocional. Barcelona: Kairós.

Goleman, D.I (2004). Inteligencia Emociona. Barcelona: Kairós.

Goleman, D. y Cherniss, C. (2005). La inteligencia emocional en el trabajo, Barcelona: Kairós.

Mayer, J. D., Brackett, M. A. y Salovey, P. (2004): Emotional intelligence: key readings on the Mayer and Salovey Model. New York: Dude Publishing.

Mayer, J. D., Salovey, P., y Caruso, D. (2000). Emotional Intelligence as Zeigeist, as Personality, and as Mental Ability. En R. Bar-On y J. D. A. Parker (Eds.), The Handbook of Emotional Intelligence. Theory, Development, Assessment, and Application at Home, School, and in the Workplace, pp. 92-117San Francisco.

Mayer, J., Salovey, P. y Caruso, D. (2000). Models of Emotional Intelligence. En R. J. Sternberg (Ed.) Handbook of Human Intelligence, pp. 396-420. New York: Cambridge University Press. 
Extremera, N. y Fernández-Berrocal, P. (2004). El uso de las medidas de habilidad en el ámbito de la Inteligencia emocional, Boletín de Psicología, 80, 59-67.

Fernández-Berrocal, P. y Extremera, N (2002). La inteligencia emocional como una habilidadesencial en la escuela, en Revista Iberoamericana de Educación, 29, 1-6. Recuperado de http://www.rieoei.org/deloslectores/326Berrocal.pdf

Salovey, P., \& Mayer, J. D. (1990). Emotional Intelligence. Imagination, Cognition and Personality, 9(3), 185-211. https://doi.org/10.2190/DUGG-P24E-52WK$\underline{6 \mathrm{CDG}}$

Sternberg, R. J. (1997). Inteligencia exitosa. Barcelona: Paidós.

Vallés Arándiga, A. y Vallés Tortosa, C. (2003): Psicopedagogía de la Inteligencia Emocional, Valencia: Promolibro. 